\title{
Lifting Objects with Power-Assist: Weight-Perception-Based Force Control Concepts to Improve Maneuverability
}

\author{
S. M. Mizanoor Rahman ${ }^{1, a}$, Ryojun Ikeura ${ }^{2, b}$, Soichiro Hayakawa ${ }^{2}$, \\ Haoyong $\mathrm{Yu}^{1}$ \\ ${ }^{1}$ Division of Bioengineering, Faculty of Engineering, National University of Singapore \\ ${ }^{2}$ Division of Mechanical Engineering, Graduate School of Engineering, Mie University, Tsu, Japan \\ amizansm@hotmail.com; bikeura@ss.mach.mie-u.ac.jp
}

\begin{abstract}
Keywords: Power Assist Robot, Lifting Objects, Human, Weight Perception, Maneuverability, Force Control, Position Control, Safe Human-Robot Interaction, Industry
\end{abstract}

\begin{abstract}
We developed a 1-DOF power assist robot system to lift objects of different sizes by human subjects. We adopted a hypothesis that weight perception due to inertia might be different from that due to gravity when lifting an object with a power assist robot because the human feels a difference between the actual weight and the perceived weight of the object. We included this hypothesis in the robot dynamics. We then discussed the suitability of force control for the robot for lifting objects and developed several weight-perception-based force control strategies. These force control strategies may be compared to previously developed position control strategies, and the comparison results may help determine appropriate control for the robot to achieve desired maneuverability. The results, as a whole, may help develop human-friendly power assist devices to handle heavy objects in various industries.
\end{abstract}

\section{Introduction}

Power assist robot systems are used mainly for health supports and rehabilitation [1], [2]. We proposed a novelty in power-assist applications in [3]. In [3], we proposed that power assist systems could be used to manipulate heavy objects in industries. Workers in industries frequently manipulate heavy objects e.g. (i) loading heavy objects (luggage, bags etc.) to aircrafts, ships, buses, trains, trucks etc., (ii) handling heavy objects, materials, bags etc. in industries such as automobile, military services, agriculture, ship building and breaking, disaster and rescue operations, mining, manufacturing and assembly, construction, timber, forestry, daily household activities etc. However, handling heavy objects manually is very hard, may cause disabilities and disorders in workers such as back pain, injuries, musculoskeletal problems etc., and on the contrary, uses of autonomous systems for handling heavy objects may not provide flexibility and satisfactory interactions with humans. Hence, human-robot systems such as power assist systems seem to be suitable to handle heavy objects/materials in the aforementioned cases. However, suitable power assist devices to handle heavy objects in industries are not so visible.

There are few models of power assist robots for objects manipulation [4], [5]. However, these models do not include human features such as human's heaviness perception in their controls, and hence, these models are not so human-friendly. We demonstrated a model of power assist system for lifting objects in [3], and included human's weight perception in the robot dynamics to improve the performances especially the maneuverability of the robotic system. The model proposed in [3] considered lifting objects in vertical direction and feedback position control was used for the system, and the position control was proven effective.

However, position control is better in some areas, but force control is better in some other areas, and the control method should be selected based on specific task requirements and on comparative results between position and force control. This paper introduced several weight-perception-based force control strategies for lifting objects with power-assist to improve maneuverability. 


\section{Materials}

We developed a 1-DOF power assist system for lifting objects as shown in Fig.1. A ball screw was actuated by a servomotor. A force sensor was tied to the ball nut and the object (rectangular thin aluminum box) was tied to the force sensor.

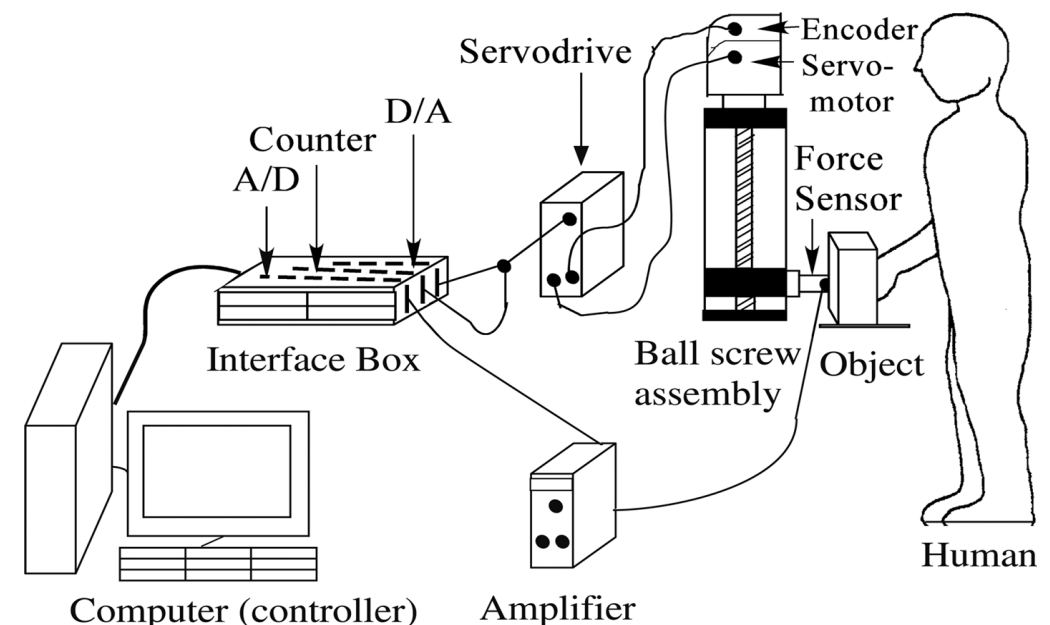

(a)

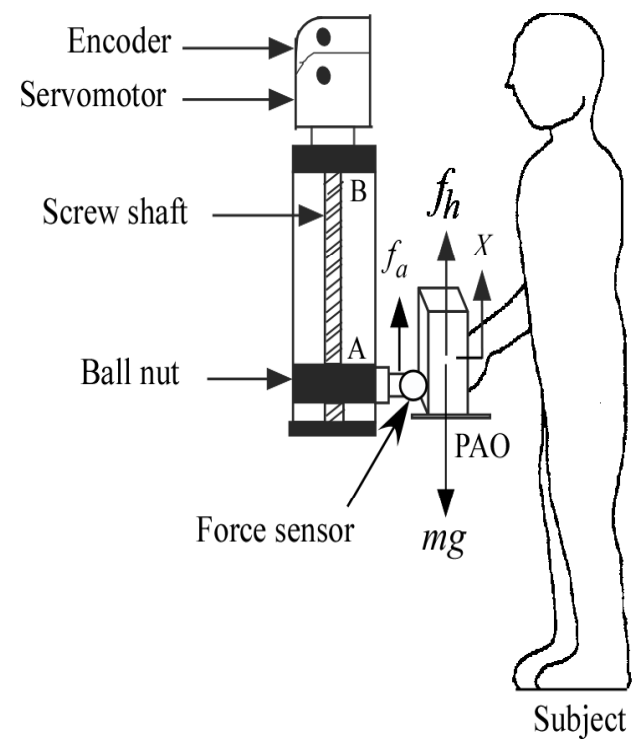

(b)

Fig.1 (a) 1-DOF power assist robot system for lifting objects. (b) Dynamics for lifting an object with

\section{Dynamics and Control} the system.

\section{Method 1:}

As shown in Fig.1, the dynamic equation for the real system is as Eq.(1), where $m$ is the actual mass of the object, $x$ is displacement, $x$ " is acceleration, $f_{\mathrm{h}}$ is human force (load force), $f_{\mathrm{a}}$ is actuator force, $g$ is acceleration of gravity. However, the model system is as in Eq.(2). We adopted a hypothesis pertaining to weight perception that perception of weight due to inertia differs from perceived weight due to gravity when manipulating an object with a power assist robot. For this reason, we thought that the mass parameter for inertia force might be different from that for gravity force for the dynamics of manipulating an object with the power assist robot [3]. Hence, Eq.(2) becomes Eq.(3). We subtract Eq.(3) from Eq.(1) and get Eq.(4) and then get Eq.(5) from Eq.(4). 


$$
\begin{aligned}
& m x "=f_{\mathrm{h}}+f_{\mathrm{a}}-m g \\
& m x "=f_{\mathrm{h}}-m g \\
& m_{1} x "=f_{\mathrm{h}}-m_{2} g \\
& \left(m-m_{1}\right) x "=f_{\mathrm{a}}-\left(m-m_{2}\right) g \\
& f_{\mathrm{a}}=\left(m-m_{1}\right) x "+\left(m-m_{2}\right) g
\end{aligned}
$$

We see in Eq.(5) that there is no $f_{\mathrm{h}}$. Hence, no force sensor is required.But, acceleration sensor is required. It is possible to measure $x$ from encoder $\&$ counter and to derive $x$ ". But it is noisy. Hence, acceleration sensor is necessary. We diagram the force control based on Eq.(5) as shown in Fig.2.

Method 2:

We multiply Eq.(1) by $m_{1}$ and Eq.(3) by $m$, and then subtract the latter from the former, and get Eq.(6). Hence, a force sensor is necessary to measure $f_{\mathrm{h}}$, but there is no need of acceleration sensor. We diagram the force control based on Eq.(6) as shown in Fig.3.

$f_{\mathrm{a}}=\left(1 / m_{1}\right)\left\{\left(m_{1}-m_{2}\right) m g-\left(m_{1}-m\right) f_{\mathrm{h}}\right\}$

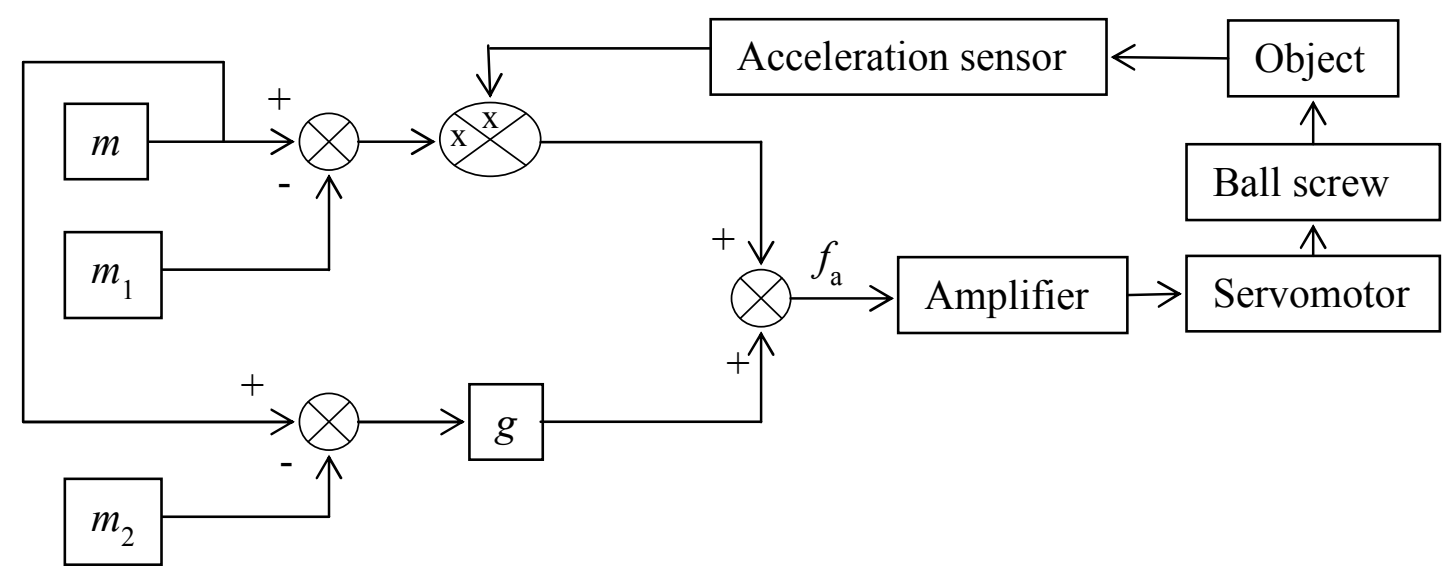

Fig.2 Block diagram of weight-perception-based force control (method 1).

\section{Experiment Methods}

We may simulate the system shown in Fig.2 and Fig.3 separately using Matlab/Simulink (solver: ode4, Runge-Kutta; type: fixed-step; fundamental sample time: $0.001 \mathrm{~s}$ ) for different values of $m, m_{1}$ and $m_{2}$ and may subjectively evaluate maneuverability for the system. Then, we may compare the maneuverabilty obtained for force control to that previously obtained for position control in [3]. The comparison results may help determine appropriate control strategies for the robot to achieve desired maneuverability.

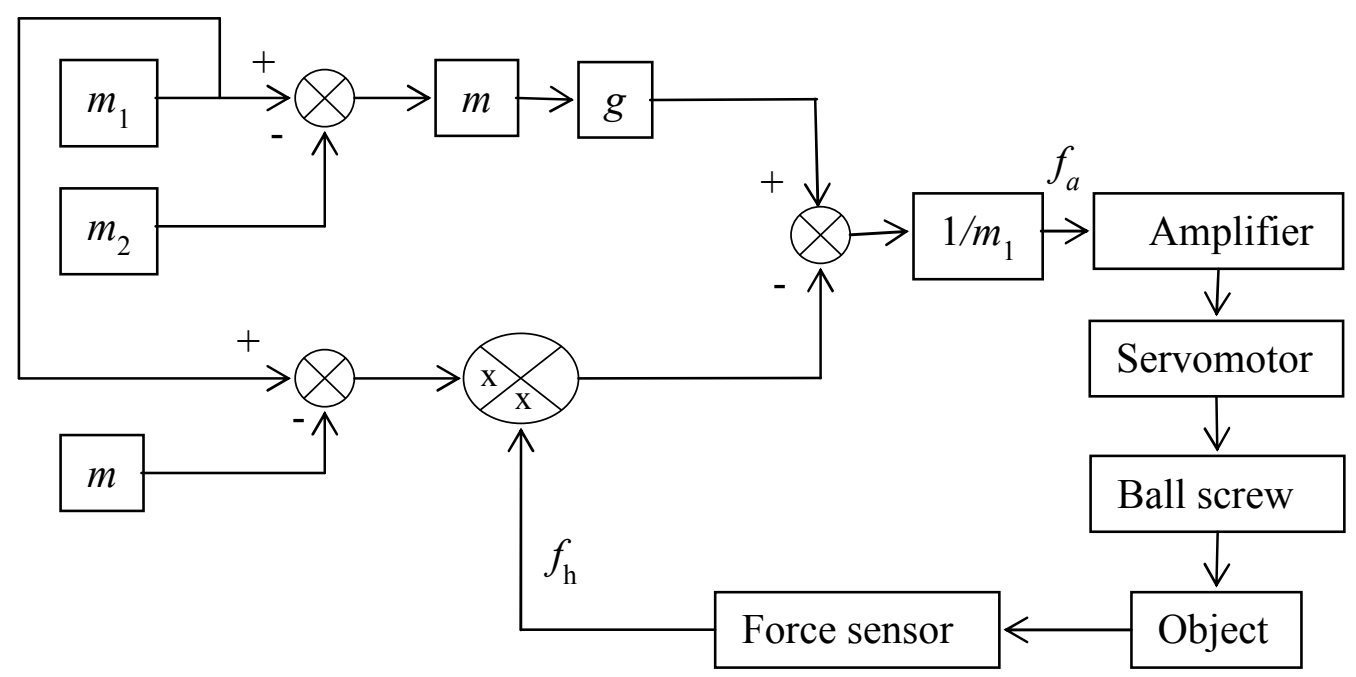

Fig.3 Block diagram of weight-perception-based force control (method 2). 


\section{Conclusions and Future Works}

We developed a 1-DOF power assist robot system for lifting objects. We included human's weight perception in the dynamics and derived force control schemes following two methods. The novelty in these force control schemes is that these schemes include human characteristics such as weight perception. We will simulate the system shown in Fig.2 and Fig.3 separately using Matlab/Simulink for different values of $m, m_{1}$ and $m_{2}$ and will subjectively evaluate maneuverability for lifting objects with the system. Then, we will compare the maneuverabilty obtained for force control to that previously obtained for position control in [3]. The comparison results may help determine appropriate control strategies for the robot system to achieve desired maneuverability. We will also compare maneuverability between horizontal and vertical manipulation of objects with the power assist robot system [6]-[9].

\section{References}

[1] H. Seki, K. Ishihara and S. Tadakuma, "Novel regenerative braking control of electric power-assisted wheelchair for safety downhill road driving," IEEE Trans. on Industrial Electronics, Vol. 56, No. 5,2009, pp. 1393-1400.

[2] S. Tashiro and T. Murakami, "Step passage control of a power-assisted wheelchair for a caregiver," IEEE Trans. on Industrial Electronics, Vol. 55, No. 4, April 2008, pp. 1715-1721.

[3] S.M.M.Rahman, R. Ikeura, M. Nobe, S. Hayakawa, H. Sawai, "Weight-perception-based model of power assist system for lifting objects," Int. Journal of Automation Technology, Vol.3, No.6, pp. 681-691, Nov 2009.

[4] T.Doi, H. Yamada, T. Ikemoto, and H. Naratani, "Simulation of a pneumatic hand crane power-assist system," Journal of Robotics and Mechatronics, Vol.20, No.6, 2008, pp. 896-902.

[5] A. Niinuma, T.Miyoshi, K. Terashima, Y. Miyashita, "Evaluation of effectiveness of a power-assisted wire suspension system compared to conventional machine," in Proc. of 2009 IEEE Int. Conf. on Mechatronics and Automation, Aug. 9-12, 2009, pp.369-374.

[6] S.M.M.Rahman, R. Ikeura, H. Sawai, "Design and control of a power-assist robot system for lifting objects based on human's unimanual and bimanual weight discrimination," International Journal of Assistive Robotics and Systems, Vol.11, No.2, pp.11-29, June 2010.

[7] S.M.M.Rahman, R. Ikeura, S. Hayakawa, and H. Sawai, "Design guidelines for power assist robots for lifting heavy objects considering weight perception, grasp differences and worst-cases," International Journal of Mechatronics and Automation, Vol. 1, No. 1, pp.46-59, March 2011.

[8] S.M.M.Rahman, R. Ikeura, S. Hayakawa, H. Sawai, "Design and control of a power assist system for lifting objects based on human operator's weight perception and load force characteristics," IEEE Transactions on Industrial Electronics, Vol.58, No.8,pp.3141-3150, August 2011.

[9] S.M.M.Rahman, R. Ikeura, M. Nobe, H. Sawai, "Study on optimum maneuverability in horizontal manipulation of objects with power-assist based on weight perception," in Proceedings of SPIE, Vol. 7500, 75000P (2010). 